\title{
Estimates of comet fragment masses from impact crater chains on Callisto and Ganymede
}

\author{
William B. McKinnon \\ Department of Earth and Planetary Sciences and McDonnell Center for the Space Sciences, \\ Washington University, Saint Louis, MO \\ Paul M. Schenk \\ Lunar and Planetary Institute, Center for Advanced Space Studies, Houston, TX
}

\begin{abstract}
Chains of impact craters, or catenae, have been identified in Voyager images of Callisto and Ganymede. Although these resemble in some respects secondary crater chains, the source craters and basins for the catenae cannot be identified. The best explanation, proposed by Melosh and Schenk, is a phenomenon similar to that displayed by former comet P/Shoemaker-Levy 9: tidal (or other) breakup close to Jupiter followed by gradual orbital separation of the fragments and collision with a Galilean satellite on the outbound leg of the trajectory. Because the trajectories must pass close to Jupiter, this constrains the impact geometry (velocity and impact angle) of the individual fragments. For the dominant classes of impactors, short-period Jupiter-family comets and asteroids, velocities at Callisto and Ganymede are dominated by jovian gravity and a satellite's orbital motion, and are insensitive to the pre-fragmentation heliocentric velocity; velocities are insensitive to satellite gravity for all impactor classes. Complex crater shapes on Callisto and Ganymede are determined from Voyager images and Schmidt-Holsapple scaling is used to back out individual fragment masses. We find that comet fragment radii are generally less than $\sim 500 \mathrm{~m}$ (for ice densities), but can be larger. These estimates can be compared with those for the Shoemaker-Levy 9 impactors.
\end{abstract}

\section{Introduction}

Understanding the Shoemaker-Levy 9 impacts requires knowledge of individual fragment masses and densities, as these critical parameters governed the spatial and temporal deposition of energy and cometary materials in the jovian atmosphere. Pre-encounter diameter predictions ranged from up to several $\mathrm{km}$ for some fragments [Weaver et al., 1994] to values an order of magnitude less [Scotti and Melosh, 1993]. Analyses of the events themselves have not yielded a consensus as of this writing. We argue here, however, that there is a historical record of similar comet fragmentations, expressed as otherwise enigmatic chains of impact craters on the Galilean satellites Callisto and Ganymede. Moreover, the trajectories that the crater-chain-forming fragments took are fairly well constrained, so by using modern crater scaling relations a reasonably definitive estimate of the masses of historical comet fragments can be obtained. We find that "large" fragments, defined here to be $>1 \mathrm{~km}$ in diameter (assuming an ice density), are not ruled out by the historical record, but would be unusual. Below we discuss the geological record left by fragmented comets, the

\section{Copyright 1995 by the American Geophysical Union.}

Paper number 95GL01422

0094-8534/95/95GL-01422\$03.00 crater shape and scaling issues that affect interpretation of this record, the probable fragment trajectories and velocities, and finally, our inversion for the fragment (and parent comet) masses and equivalent radii, and implications.

\section{Crater Chains}

Distinctive crater chains (or catenae) have been identified on Callisto, and subsequently, Ganymede. They are remarkably linear and unrelated to underlying geology (Figure 1; Schenk et al., manuscript in preparation, 1995). At least 8 such chains have been identified on Callisto, 3 on Ganymede. Several linear chain-like features also exist, but at resolutions too poor to classify with confidence. Catenae craters on Callisto have well-developed raised rims and central peaks (or pits) (Fig. 1a), and two of the three recently detected chains on Ganymede [Melosh and Schenk, 1993] show unequivocal ejecta deposits, all strong evidence for an impact origin. Supporting evidence includes possible associated ejecta on Callisto (ejecta being more difficult to discern on Callisto's less ice-rich surface) (Fig. 1a), chains that cross preexisting structures or structural boundaries ((i.e., basin rings, cratered terrain/grooved terrain) uninterrupted or influenced, and overlapping craters within several catenae (e.g., Figure lb).

Among known impact features, the catenae best resemble secondary crater chains such as those radiating from large lunar basins [Wilhelms, 1987] or the Gilgamesh basin on Ganymede. Passey and Shoemaker [1982] originally judged the chains on Callisto to be secondary craters of collapsed or relaxed basins that had disappeared from the geological record, despite the high stratigraphic positions of some of the chains, but the search for possible source craters and basins did not meet with success [McKinnon, 1983]. Fragments of impactors striking one satellite at grazing incidence and then traveling on to the next have also been suggested [Halfen et al., 1990]. Whether or not this explanation works physically, the geometric cross-section ricochet trajectories must thread
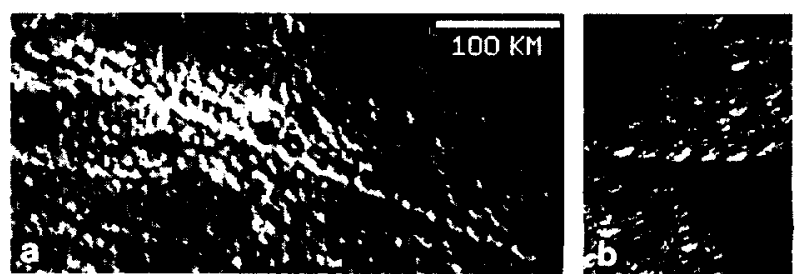

Figure 1. Voyager images of two crater chains on Callisto; same scale, north up. (a) 25 -crater chain on the northern portion of the Valhalla multiringed system, centered at $35^{\circ} \mathrm{N}$. $47^{\circ} \mathrm{W}$ (from FDS 16424.32, rectified); (b) 7-crater chain, centered at $49^{\circ} \mathrm{N}, 348^{\circ} \mathrm{W}$ (from FDS 16426.10 , rectified). 
through, and thus their probability, is substantially smaller than that appropriate to comets that break up near Jupiter and subsequently strike a satellite.

The remarkable similarity of the Shoemaker-Levy 9 "string of pearls" led Melosh and Schenk [1993] to argue that the catenae on Callisto and Ganymede represent a fossil record of similar comet splitting events within the Jovian system, whether due to tidal stresses [e.g., Melosh and Schenk, 1993; Scotti and Melosh, 1993] or, possibly, to collisions with ring particles [Asphaug and Benz, 1994]. We adopt break-up near Jupiter as the initial condition for the formation of catenae.

\section{Crater Geometry and Scaling}

In order to determine the masses of the impactors that made the catenae craters, the mass excavated must be determined for each crater. Catenae craters range from 5 to $40 \mathrm{~km}$ in diameter (measured perpendicular to the catena axis when necessary to avoid overlap), and all (or nearly all) are morphologically complex, or collapsed. To determine the mass excavated, the transient, pre-collapse shape must be derived from the final, observed shape for each. Shape cannot be measured reliably for catenae craters on Callisto or Ganymede. None are close enough to the terminator to use shadows, and strong albedo variations on Callisto make photoclinometric (shape from shading) slope estimates there unreliable [Schenk, 1991]. Because of the strong similarity of catenae crater morphology to that of complex craters overall on Ganymede and Callisto, we use the general crater morphological statistics of Schenk [1991] to describe crater shapes.

We model complex craters as "pie tins," with flat floors (central peaks and pits have negligible volumes for our purposes), steep inner walls, and raised rims. Photoclinometric and shadow-length measurements of (normal) complex crater depths $(d)$ and rim heights $(h)$ on Ganymede from Schenk [1991] can be fit with power laws of the form $d=$ $0.26( \pm 0.05) D_{\mathrm{f}}^{0.39 \pm 0.06}$ and $h-0.09 D_{\mathrm{f}}^{0.33}$, where $D_{\mathrm{f}}$ is final crater diameter and all units are in $\mathrm{km}$. The smaller set of shadow-length measurements of crater depths on Callisto are consistent with the Ganymede data, so because the geology and gravity on the two satellites are similar, we use the Ganymede fits for both. Inner rim slopes are taken at a constant $25^{\circ}$ and outer rim flanks at $8^{\circ}$, an approximation, but one consistent with photoclinometric profiles [e.g., Schenk, 1993]. We assume volume conservation during the modification stage and neglect distributed rim subsidence, and model the transient geometry as a paraboloid of revolution, extending the rim (at $8^{\circ}$ ) until a total depth/diameter of $1 / 2 \sqrt{2}$ [e.g., Grieve and Garvin, 1984 ] is achieved. This results in an approximate law relating transient to final crater diameters on Ganymede and Callisto:

$$
D_{\mathrm{f}} \sim 1.176 D_{\mathrm{tr}^{1}}^{1.108}
$$

where $D_{\mathrm{tr}}$ is the transient diameter. This relationship is probably accurate to $-15 \%$, and the excavated volumes (and masses) to $-50 \%$. Equation (1) predicts that final craters in the $5-40-\mathrm{km}$ diameter range are $\sim 40-60 \%$ wider than their transient forms, substantially greater than the $30 \%$ enlargement assumed by Shoemaker and Wolfe [1982] in their treatise on cratering rates in the Jupiter system. Their enlargement factor was based on studies of collapsed lunar craters, but complex craters on Ganymede and Callisto are much flatter than lunar craters (which was not known in 1982), having collapsed to a greater degree [Schenk, 1991, 1993]. The exponential dependence in equation (1) is, however, similar to that derived for lunar complex craters [see McKinnon et al., 1991; Holsapple, 1993].

Once the transient crater volume $V$ is determined, the impactor mass and radius $a$ can be determined from scaling laws for a selected impact velocity $u$ and impact angle from vertical $\theta$, when $u$ is replaced by $u \cos \theta$ [Chapman ana McKinnon, 1986]. Schmidt-Holsapple scaling is used (see Holsapple [1993]). Cratering efficiency (mass excavated divided by impactor mass) is determined as a function of gravity-scaled size $\pi_{2}=g a / u^{2}$ for impacts into ice in both the strength and gravity regimes ( $g$ is surface gravity). The effective strength $\bar{Y}$ of ice is taken to be that used by Holsapple [1993] to model impacts into soft rock (see his figure 6), and is thus approximate; the surface density is assumed to be $1 \mathrm{~g} / \mathrm{cm}^{3}$. Nearly all catenae craters form in the gravity regime, based on the range of fragment impactor sizes derived below and the impact velocities at Callisto and Ganymede $\left(\pi_{2}\right.$ lies between $\sim 3 \times 10^{-7}$ and $10^{-5}$ ); hence the exact scaling in the strength regime is not so important. The absolute calibration of the cratering efficiency in the gravity regime remains uncertain, however, perhaps by a factor of 2 (based on the range of previously published estimates). This dominates the overall uncertainty in our calculations.

\section{The Impactor Flux at Jupiter}

Impacts on Callisto and Ganymede are caused by comets and asteroids. Of these, short-period comets and asteroids on shortperiod-comet-like orbits (extinct comets) dominate the flux; Halley-family and long-period comets contribute perhaps $10 \%$ [Shoemaker et al., 1986, Shoemaker, 1994]. The orbits of 97 short-period comets discovered through 1978, along with synthetic model orbits, were used by Shoemaker and Wolft [1982] to determine a weighted encounter velocity $v_{\infty}=4.5$ $\mathrm{km} / \mathrm{s}$ at Jupiter's gravitational sphere of influence, only $\sim 0.35$ of Jupiter's mean orbital speed $v_{\text {circ }} \approx 13 \mathrm{~km} / \mathrm{s}$. Gravitational focusing reduces this relative velocity for close encounters. Long-period comets, while rarer, encounter Jupiter at much higher speeds $v_{\infty} \approx \sqrt{3} v_{\text {circ }} \approx 22.5 \mathrm{~km} / \mathrm{s}$. New discoveries of extinct comets (asteroids) include higher inclination objects [Shoemaker, 1994], which increase their weighted $v_{\infty}$. P.R. Weissman [personal communication, 1994] recommends values closer to $0.6 v_{\text {circ }} \approx 8 \mathrm{~km} / \mathrm{s}$. At the risk of maximizing the derived impactor masses, we will adopt a value at the low end of the spectrum, $v_{\infty}=0.3 v_{\text {circ }} \approx 3.9 \mathrm{~km} / \mathrm{s}$, for short-period comets and dynamically similar asteroids or extinct comets. While the probability distribution of $v_{\infty}$ for short-period comets is uncertain, we shall see that it is not the dominant determinant of collision speeds on Callisto and Ganymede, and hence not the dominant source of uncertainty in our results.

\section{Fragment Trajectories}

The trajectorics in Figure 2 illustrate encounters of shortperiod comets with $v_{\infty}=0.3 v_{\text {circ }}$ that pass through Jupiter's Roche zone, 1 10 $\approx 2.5\left(\rho_{\mathrm{J}} / \delta\right)^{1 / 3} R_{\mathrm{J}}$, where $R_{\mathrm{J}}$ and $\rho_{\mathrm{J}}$ are Jupiter's radius and density $\left(=1.33 \mathrm{~g} / \mathrm{cm}^{3}\right)$, respectively, and $\delta$ is the cometary (or asteroidal) density, although tidal break-up may only occur within $\approx 1.7\left(\rho_{\mathrm{J}} / \delta\right)^{1 / 3} R_{\mathrm{J}}[$ Sridhar and Tremaine, 1992]. Outbound the trajectories pass through Callisto's or Ganymede's position at either the ascending or descending node on the Jovian equatorial plane. Based on 2body (Jupiter+comet) hyperbolic orbits, the angle the trajectories make with respect to the radius vector to Jupiter is constrained, $\sim 13^{\circ}$ at Callisto and $\sim 18^{\circ}$ at Ganymede for $r_{\min } \approx$ $1.5 R_{\mathrm{J}}$; variations of several degrees are possible depending on $r_{\min }$ and the influence of Jupiter's higher gravitational harmonics $\left(J_{2}\right.$ and $\left.J_{4}\right)$. With each satellite's motion (aberration) taken into account, projections of fragment impact trajectories shift somewhat onto the leading hemisphere of each satellite.

Comet velocities $\left(v_{c}\right)$ at each satellite's orbital distance are given by the root-sum-square of $v_{\infty}$ and the escape velocity 


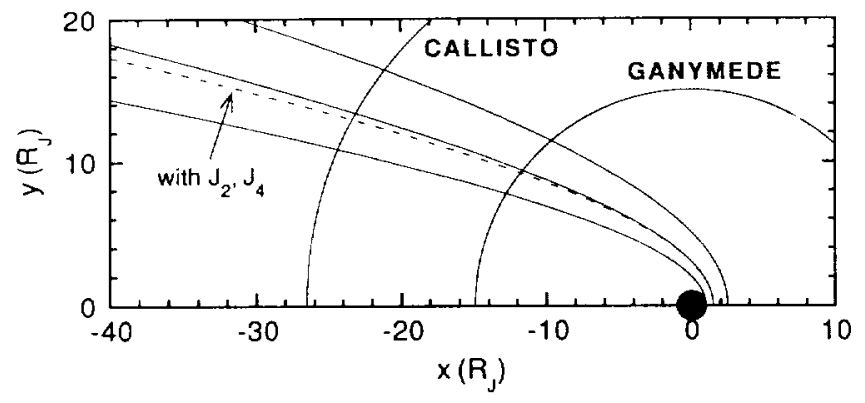

Figure 2. Trajectories in Jupiter's equatorial plane for comet fragments with $v_{\infty} \approx 0.3 v_{\text {circ, }}$ from 3 -body (Sun+Jupiter+comet) integrations of the equations of motion (courtesy L.A.M. Benner). Perijove distances are 1, 1.5, and $2.5 R_{\mathrm{J}}$. The influence of $J_{2}$ and $J_{4}$ is illustrated and is maximized for equatorial plane trajectories.

from Jupiter at the orbital distance $\left(\sqrt{ } 2 v_{\mathrm{sat}}\right)$, where $v_{\mathrm{sat}}$ is the satellite's mean orbital speed; for the 2-body problem $v_{c}$ is independent of $r_{\min }$. Because fragment trajectories are nearly perpendicular to the satellite orbits, the approach velocity to a satellite is close to the root-sum-square of $v_{c}$ and $v_{\text {sat }}$. Further combined with a given satellite's escape velocity $\left(v_{e s c}\right)$, the impact speed $u$ of a fragment on Ganymede and Callisto is

$$
u \approx\left(3 v_{\mathrm{sat}}^{2}+v_{\infty}^{2}+v_{\mathrm{esc}}^{2}\right)^{1 / 2}
$$

Escape velocity from Jupiter at Callisto's and Ganymede's radial distance is 11.6 and $15.4 \mathrm{~km} / \mathrm{s}$, respectively; $v_{\mathrm{esc}}<3$ $\mathrm{km} / \mathrm{s}$ for the satellites themselves. Hence, for $v_{\infty}=0.3 v_{\text {circ }}$ $\approx 4 \mathrm{~km} / \mathrm{s}, u$ is dominated by jovian gravity and satellite motion, and $u \approx 15$ and $19.5 \mathrm{~km} / \mathrm{s}$ for Callisto and Ganymede, respectively. Even for $v_{\infty}=0.6 v_{\text {circ }}$, the depth of the Jovian gravitational well is such that $u$ only changes by $10 \%$ at Callisto and $6 \%$ at Ganymede. Only for high-speed, long-period comets does $v_{\infty}$ become critically important, if not dominant.

The wire frames in Figure 3 illustrate the locations of all the probable crater chains; five small chains in the original compilation of Melosh and Schenk [1993] are not included because their impact origin is equivocal. The projections are centered on the equator near $35^{\circ}$ and $36^{\circ} \mathrm{W}$, normal to the average of the projected trajectory vectors for Callisto and Ganymede, respectively (for $r_{\mathrm{min}} \approx 1.5 R_{\mathrm{J}}$ and $v_{\infty} \approx$ $\left.0.3 v_{\text {circ }}\right)$. All of the observed chains are located on the appropriate (tidally fixed) hemisphere of each satellite. The projected trajectory vector of an individual comet (its velocity relative to a satellite) depends on the inclination of that comet's trajectory with respect to the Jovian equator. As the inclination varies from prograde (direct) to retrograde, the position on a satellite's surface where the trajectory vector is normal to the surface traces out an oval, for fixed $r_{\min }$ and $v_{\infty}$. These ovals are shown in Figure 3 for $r_{\min }=1.5 R_{\mathrm{J}}$ and $v_{\infty}=0.3 v_{\text {circ. }}$. The direct and retrograde trajectories in Jupiter's equatorial plane are normal to a satellite's surface, and the cometary velocity minimized and maximized, at the easternmost and westernmost points on the ovals, respectively. Gravitational focusing of the trajectories by the satellites themselves is minimal (the cross-sections are altered by only $1-2 \%$ ) and is neglected in this calculation and below.

The inclination of a trajectory also determines the orientation of a chain on the satellite, with two possible inclinations for each orientation. Impact sequence is the key to choosing among the two (see Figure lb), but this cannot as yet be done for most of the chains in Figure 3. Thus, for this letter we simply assume that $u$ is given by the mean values above, and that $\theta$ for each chain crater is the angular distance from the crater to the center of the appropriate oval in Figure 3. This introduces an uncertainty in $u$ of $\sim 10 \%$ and in $\theta$ of $\sim 10^{\circ}$ to $15^{\circ}$ for catenae on Callisto and Ganymede, respectively. Impact angle enters the scaling through $\cos \theta$, however, so when $\theta$ is small its uncertainty is of minor consequence.

\section{Fragment Masses and Radii}

The comet fragment masses as determined from the crater scaling and trajectory estimates above are shown in Figure 4 with the Callisto and Ganymede data identified. The Callisto and Ganymede contributions are mutually consistent. Fragment mass estimates for all observed catenae craters range over 4 orders of magnitude, with a concentration near the center of the distribution. In contrast, mass estimates for individual catenae are limited in range to approx. 1 order of magnitude, and some are more nearly equal. Specifically, catenae whose number of craters best compares with the Shoemaker-Levy fragment train have a mass range of an order of magnitude. Thus, fragment masses vary from comet to comet, but individual comets tend to split into more similar mass fragments. This would be consistent with tidal breakup models in which gravitational instabilities cause roughly equal mass accumulations [Asphaug and Benz, 1994; Solem, 1994], but not with theories that predict a characteristic size or size-spectrum of comet building blocks [Weidenschilling, 1994], unless the characteristic size(s) are much smaller than the fragments. While Gipul Catena and a shorter chain on Ganymede are the sole contributors to the high end of the mass distribution, without them the total range in fragment mass is still $10^{3}$.
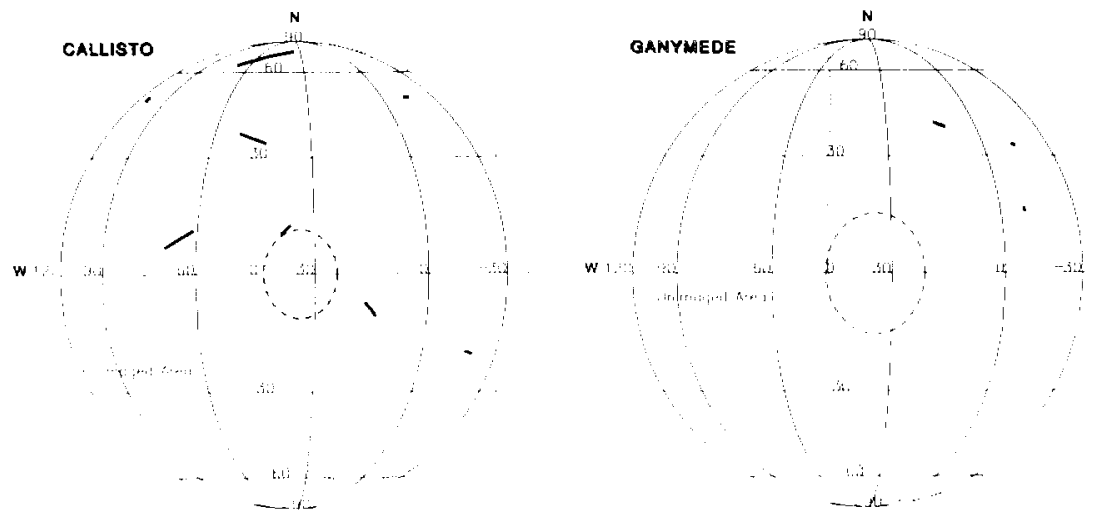

Figure 3. View of Callisto and Ganymede centered on the average trajectory vector for $v_{\infty} \approx 0.3 v_{\text {circ }}$ and $r_{\mathrm{min}}=1.5 R_{\mathrm{J}}$. Crater chains are shown, as are loci of trajectory normal vectors (see text) and imaging limits. 

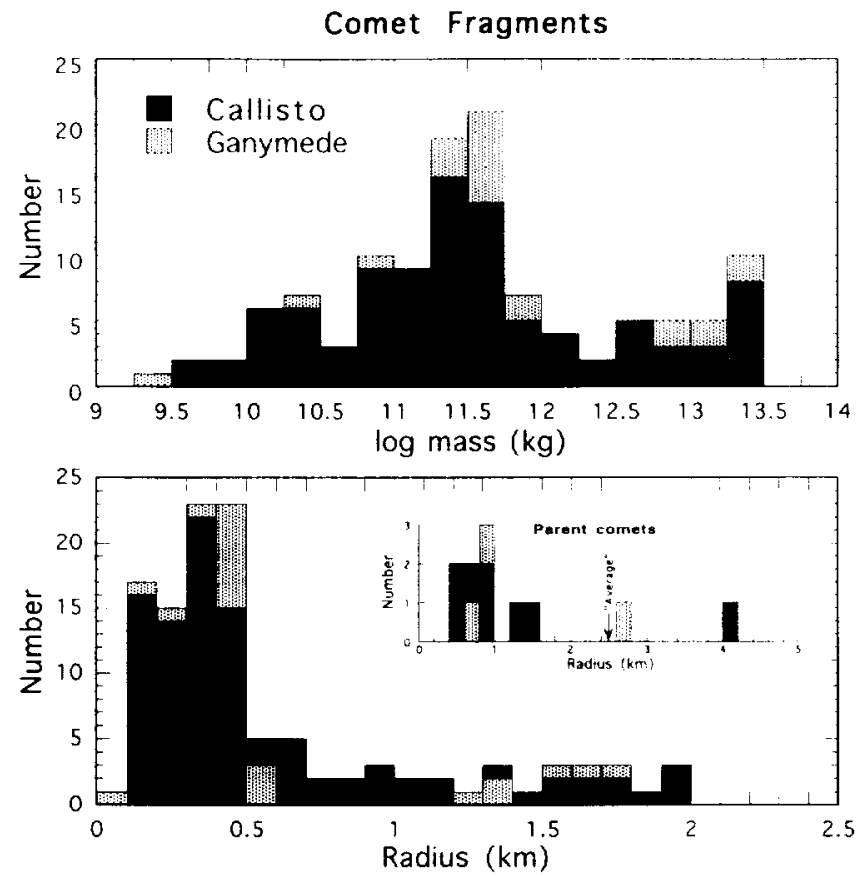

Figure 4. Histograms of comet fragment masses and radii and (inset) parent comet radii. Radii assume a density of 1 $\mathrm{g} / \mathrm{cm}^{3}$. "Average" comet radius from Weissman [1991] is for comets brighter than absolute magnitude 11 .

Equivalent fragment radii in Figure 4 are calculated assuming an ice density $\left(1 \mathrm{~g} / \mathrm{cm}^{3}\right)$. Most fragment radii are small, $\sim 100-700 \mathrm{~m}$, and less than many estimates of the Shoemaker-Levy 9 fragments. The distribution does have an extended tail to larger sizes, however, giving a median of -400 $\mathrm{m}$ and a mean of $\sim 600 \pm 500 \mathrm{~m}$. The low end may be undersampled, but the resolution on substantial portions of the satellites is good enough to pick out smaller catenae, if they were abundant.

Fragments larger than $1 \mathrm{~km}$ radius are found only for the largest catena on each satellite. If the Shoemaker-Levy 9 fragments were large ( $>1 \mathrm{~km}$ diameter), the event would be historically unusual. Of course, noticing the comet may itself illustrate a selection effect; SL-9 may have been one of the historically larger, more massive comets. We note that adopting a different density for the fragments, e.g., $0.6 \mathrm{~g} / \mathrm{cm}^{3}$, increases fragment size by a factor of 1.2 , and if the satellite surfaces are denser than ice, fragment masses go up nearly in proportion. The total (mostly systematic) uncertainty in fragment mass may be a factor of $\sim 3$, or $\sim 1.4$ in radius.

Reconstructed radii for the parent comets are also shown in Figure 4. Few comet radii and no comet masses have been measured directly, but our estimates overlap those in Weissman [1991]. Future work will involve more detailed examination of split comet trajectories and catena geology (Schenk et al., manuscript in preparation, 1995), and beginning in 1996, new imagery from the Galileo Orbiter.

Acknowledgments. We thank Lance Benner and two anonymous referees. This research was supported by NASA Planetary Geology and Geophysics Program grant NAGW-432, and is Lunar and Planetary Institute contribution no. 861.

\section{References}

Asphaug, E., and W. Benz, Density of comet Shoemaker-Levy 9 deduced by modelling breakup of the parent 'rubble pile,' Nature, 370, 120-124, 1994.

Chapman, C.R., and W.B. McKinnon, Cratering of planetary satellites, in Satellites, edited by J.A. Burns and M.S. Matthews, pp. 492-580, Univ. of Arizona Press, Tucson, 1986.

Grieve, R.A.F., and Garvin, J.B., A geometric model for the excavation and modification at terrestrial simple impact craters, J. Geophys. Res., 89, 11561-11572, 1984.

Halfen, C.W., P.H. Schultz, and D.E. Gault, Origin of anomalous crater chains and their implications for the cratering record, Lunar Planet. Sci. XXI, 447-448, 1990.

Holsapple, K.A., The scaling of impact processes in planetary sciences, Ann. Rev. Earth Planet. Sci., 21, 333-373, 1993.

McKinnon, W.B, Impact crater chains on Callisto, and the origin of the S, N, and C meteorites, EOS Trans. AGU, 64, 254, 1983.

McKinnon, W.B, C.R. Chapman, and K.R. Housen, Cratering of the Uranian satellites, in Uranus, edited by J. Bergstralh, E.T. Miner and M.S. Matthews, pp. 629-692, Univ. of Arizona Press, Tucson, 1991

Melosh, H.J. and P. Schenk, Split comets and the origin of crater chains on Ganymede and Callisto, Nature, 365, 731-733, 1993

Passey, Q.R., and E.M. Shoemeker, Craters and basins on Ganymede and Callisto: Morphological indicators of crustal evolution, in Satellites of Jupiter, edited by D. Morrison, pp. 379-434, Univ. of Arizona Press, Tucson, 1982.

Scotti, J.V., and H.J. Melosh, Estimate of the size of comet Shoemaker-Levy 9 from a tidal breakup model, Nature, 365, $733-735,1993$.

Schenk, P.M., Ganymede and Callisto: Complex crater formation and planetary crusts, $J$. Geophys. Res., 96, 15,635-15,664, 1991

Schenk, P.M., Central pit and dome craters: Exposing the interiors of Ganymede and Callisto, J. Geophys. Res., 98, 7475-7498, 1993.

Shoemaker, E.M., Update on the impact rates in the Jovian system, in $\mathrm{ky}$ Galilean Satellites Conf. abs., San Juan Capistrano Res. Inst., 71, 1994.

Shoemaker, E.M., and R.F. Wolfe, Cratering time scales for the Galilean satellites, in Satellites of Jupiter, edited by D. Morrison, pp. 277-339, Univ. of Arizona Press, Tucson, 1982.

Shoemaker, E.M., R.F. Wolfe, and C.S. Shoemaker, Extinct Jupiter-family comets and cratering rates on the Galilean satellites, Lunar Planet. Sci. Conf. XVII, 799-800, 1986.

Solem, J.C., Density and size of comet Shoemaker-Levy 9 deduced from a tidal breakup model, Nature, 370, 349-351, 1994.

Sridhar, S, and S. Tremaine. Tidal disruption of viscous bodies, Icarus, 95, 86-99. 1992.

Weaver, H.A., and 18 others, Hubble Space Telescope observations of comet P/Shoemaker-Levy 9 (1993e), Science. $263,787-791,1994$

Weidenschilling, S.J., Origin of cometary nuclei as 'rubble piles,' Nature, 368,721-723, 1994.

Weissman, P.R. The cometary impactor flux at the Earth, in Global Catastrophes in Earth History, edited by V. Sharpton and P. Ward, pp. 171-180, Geological Society of America Special Paper, 247. 1991

Wilhelms, D.E., The geological history of the Moon, U.S Geological Survey Prof. Paper, 1348, 302 pp., 1987.

W.B. McKinnon, Department of Earth and Planetary Sciences, Washington University, Saint Louis, MO 63130 (e-mail: mckinnon@wunder.wustl.edu)

P.M. Schenk, Lunar and Planetary Institute, 3600 Bay Area Blvd., Houston, TX 77058. (e-mail: schenk@lpi.jsc.nasa.gov)

(Received December 27, 1994; accepted March 28, 1995) 